\title{
$F$-region Pedersen conductivity deduced using the TIMED/GUVI limb retrievals
}

\author{
H. Kil, M. DeMajistre, L. J. Paxton, and Y. Zhang \\ Applied Physics Laboratory, Johns Hopkins University, 11100 Johns Hopkins Road, Laurel, MD 20723, USA \\ Received: 17 August 2005 - Revised: 22 December 2005 - Accepted: 9 January 2006 - Published: 3 July 2006 \\ Part of Special Issue “The 11th International Symposium on Equatorial Aeronomy (ISEA-11), Taipei, May 2005”
}

\begin{abstract}
As a proxy of the Rayleigh-Taylor instability growth rate for equatorial plasma bubbles, we investigate the flux-tube integrated $F$-region Pedersen conductivity $\left(\Sigma_{P}^{F}\right)$ using the electron density profiles (EDPs) provided by the Global Ultraviolet Imager (GUVI) on board the Thermosphere Ionosphere and Mesosphere Energetics and Dynamics (TIMED) satellite. The investigation is conducted using the EDPs obtained in the Atlantic sector at 19:00-22:00 LT during 4-17 August and 6-16 December 2002. The seasonal difference of the strength and location of the equatorial ionization anomalies (EIAs) induces a significant difference in the deduced $\Sigma_{P}^{F}$. Much stronger EIAs are created at higher altitudes and latitudes in December rather than in August. At 19:00-20:00 LT, the peak value of the $\Sigma_{P}^{F}$ has 23 mhos at $1100 \mathrm{~km}$ apex height during 14-16 December and 18 mhos at $600 \mathrm{~km}$ during $15-17$ August. The $\Sigma_{P}^{F}$ decreases as local time progresses. Therefore, $\Sigma_{P}^{F}$ provides a preferred condition for the growth of bubbles to higher altitudes at 19:0020:00 LT than at later hours, in December rather than in August in the Atlantic sector.
\end{abstract}

Keywords. Ionosphere (Equatorial ionosphere; Ionospheric irregularities; General or miscellaneous)

\section{Introduction}

The equatorial electron density irregularities in the $F$ region, often called bubbles, are known to be produced by the gravity induced collisional Rayleigh-Taylor (R-T) instability (Kelley, 1989). Modulation of the bottomside $F$ layer induces a polarization electric field, by which the low-density bottomside plasma is transported to the topside and creates irregularities. The global distribution of the irregularities shows large seasonal and longitudinal variations. The two competing hypotheses are that the bubble distribution is controlled by the bottomside seed perturbations (e.g. Röttger, 1981; McClure

Correspondence to: $\mathrm{H}$. Kil

(hyosub.kil@jhuapl.edu) et al., 1998) and by the growth condition of the instability (e.g. Abdu et al., 1981, 1992; Maruyama and Matuura, 1984; Tsunoda, 1985; Mendillo et al., 1992; Kil et al., 2004a). The growth rate of the instability depends on various factors in the $E$ and $F$ regions and also in the thermosphere. The studies related to the growth rate can be categorized into the $E$ region conditions (Abdu et al., 1981, 1992; Tsunoda, 1985) and the $F$-region conditions (Maruyama and Matuura, 1984; Mendillo et al., 1992; Kil et al., 2004a). These hypotheses were established more than two decades ago but they have not yet been validated, owing to a lack of global observation data.

The Global Ultraviolet Imager (GUVI) on board the Thermosphere Ionosphere Mesosphere Energetics and Dynamics (TIMED) satellite provides reliable electron density profiles (EDPs) in the altitude range of $200-500 \mathrm{~km}$ (Christensen et al., 2003; DeMajistre et al., 2004; Paxton et al., 2004). The equatorial ionization anomaly (EIA) is normally located in this altitude range. GUVI provides global EDPs and, for the first time, enables the investigation of the global bubble growth conditions. Kil et al. (2004a) reported close similarities in the global bubble distribution and the $F$-region plasma distribution. They postulated the use of the $F$-region Pedersen conductivity $\left(\Sigma_{P}^{F}\right)$ as a measure of the $F$-region effect on the growth of the R-T instability. We extend the study of Kil et al. by calculating $\Sigma_{P}^{F}$ using the GUVI EDPs. In Sect. 2, we describe the linear growth rate of the R-T instability. In Sect. 3, the GUVI disk and limb scan images of the OI 135.6-nm radiance and the Pedersen conductivities deduced from the GUVI EDPs are presented. Conclusions are given in Sect. 4.

\section{Linear growth rate of the R-T instability}

The linear growth rate of the flux-tube integrated R-T instability $(\Gamma)$ (Sultan, 1996) is given by

$$
\Gamma=\frac{\Sigma_{\mathrm{P}}^{\mathrm{F}}}{\Sigma_{\mathrm{P}}^{\mathrm{E}}+\Sigma_{\mathrm{P}}^{\mathrm{F}}}\left(\mathrm{V}+\mathrm{U}_{\mathrm{n}}+\frac{\mathrm{g}}{\nu_{\text {in }}^{\text {eff }}}\right) \frac{1}{\mathrm{~L}_{\mathrm{n}}}-\mathrm{R}_{\mathrm{T}} .
$$

Published by Copernicus GmbH on behalf of the European Geosciences Union. 


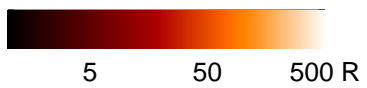

August 3-4, 2002
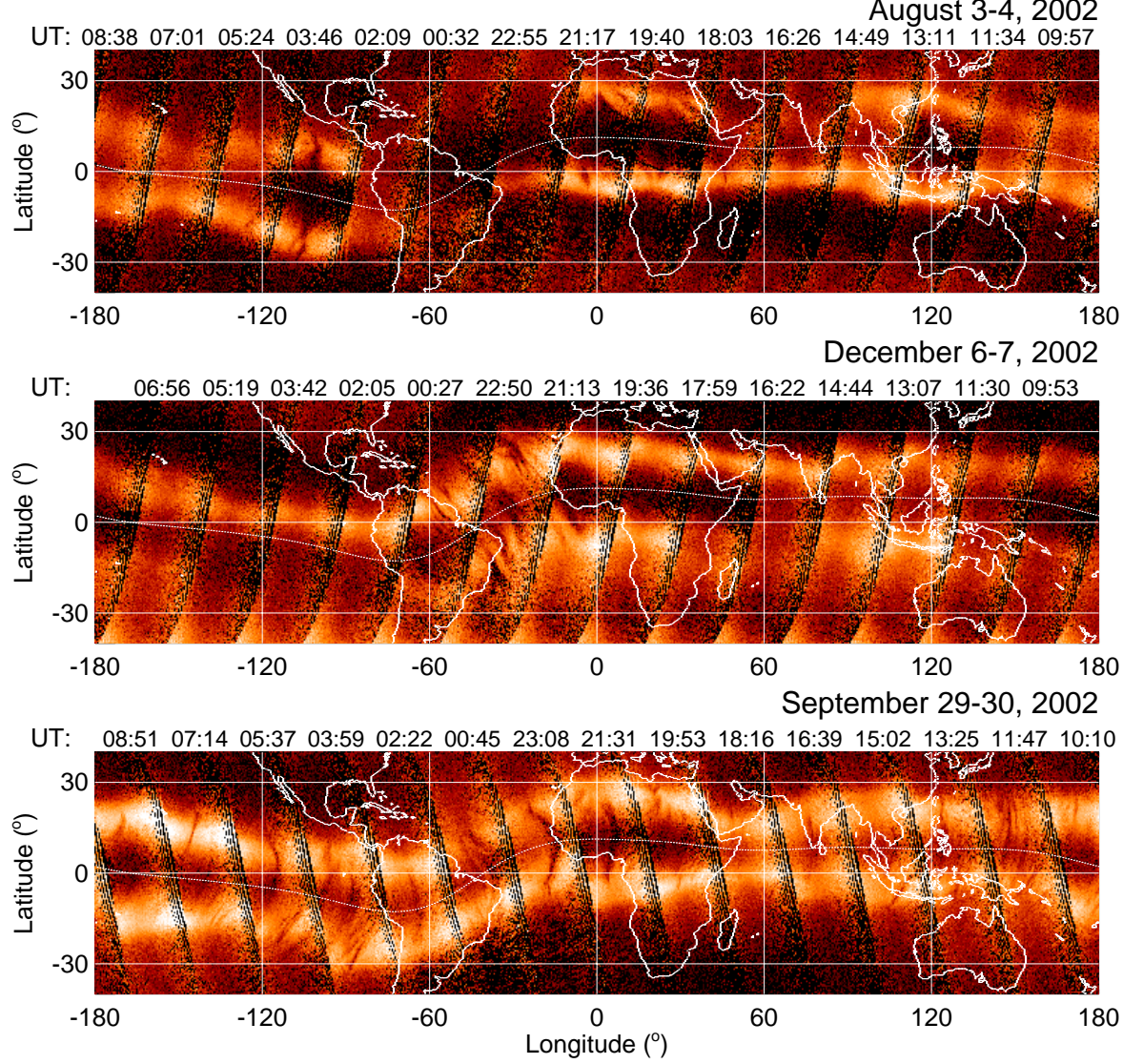

Fig. 1. Composite OI 135.6-nm radiance maps at 21:00 LT on 3-4 August (top), 6-7 December (middle), and 29-30 September (bottom) in 2002 . The bright arcs are produced by the EIAs. The dark bands within each swath of GUVI data result from plasma depletions.

Here $\Sigma_{P}^{E}$ and $\Sigma_{P}^{F}$ are flux-tube integrated $E$ - and $F$-region Pedersen conductivities; $V$ is the upward component of the plasma drift velocity, $U_{n}$ is the downward component of the neutral wind velocity, $g$ is gravitational acceleration, $v_{i n}^{\text {eff }}$ is the flux-tube integrated effective $F$-region ion-neutral collisional frequency, $L_{n}$ is the scale-length of the vertical gradient of the $F$-region flux-tube integrated plasma density, and $R_{T}$ is the flux-tube integrated recombination rate. We do not know yet which parameter plays the dominant role in the growth of the R-T instability. The parameters related to the $E$-region, such as $\Sigma_{P}^{E}$, are extremely difficult to obtain even in a local region. Our approach is to start the investigation of the growth condition of the R-T instability with the easily observable parameters. As we will discuss below, we have chosen this approach because we have noticed what appears to be a strong correlation between the intensity of the EIA observed by GUVI and the occurrence of ionospheric bubbles seen as depletions in the GUVI 135.6-nm images. Thus, it would seem reasonable to suggest that if the seasonal and longitudinal variations of $\Sigma_{P}^{F} /\left(\Sigma_{P}^{E}+\Sigma_{P}^{F}\right)$ are much larger than the variations of the quantities in the parenthesis or $L_{n}$ in Eq. (1), we can produce the global map of $\Gamma$, even though we do not know the magnitude of the other parameters. We can proceed with this line of investigation using just $\Sigma_{P}^{F}$ if the variation of $\Sigma_{P}^{F}$ is much more significant than $\Sigma_{P}^{E}$.

The flux tube integrated Pedersen conductivity $\Sigma_{P}$ is defined by (Haerendel et al., 1992)

$\Sigma_{\mathrm{P}}=\mathrm{R}_{\mathrm{E}} \mathrm{L} \int \sigma_{\mathrm{P}}\left(1+3 \xi^{2}\right) \mathrm{d} \xi$

Here $R_{E}$ is the Earth radius, $L$ is the radius of the equatorial crossing point of the field line measured in units of Earth radius, $\sigma_{P}$ is the local Pedersen conductivity, $\xi=\sin \varphi$, and $\varphi$ is the dipole latitude. The local Pedersen conductivity $\sigma_{P}$ is defined by

$\sigma_{\mathrm{P}}=\frac{\mathrm{ne}}{\mathrm{B}}\left(\frac{\kappa_{\mathrm{i}}}{1+\kappa_{\mathrm{i}}^{2}}-\frac{\kappa_{\mathrm{e}}}{1+\kappa_{\mathrm{e}}^{2}}\right)$

with the symbols $n$ for the electron density, $e$ for the elemental charge, $B$ for the magnetic field, and $\kappa_{j}$ for the ratio of the 

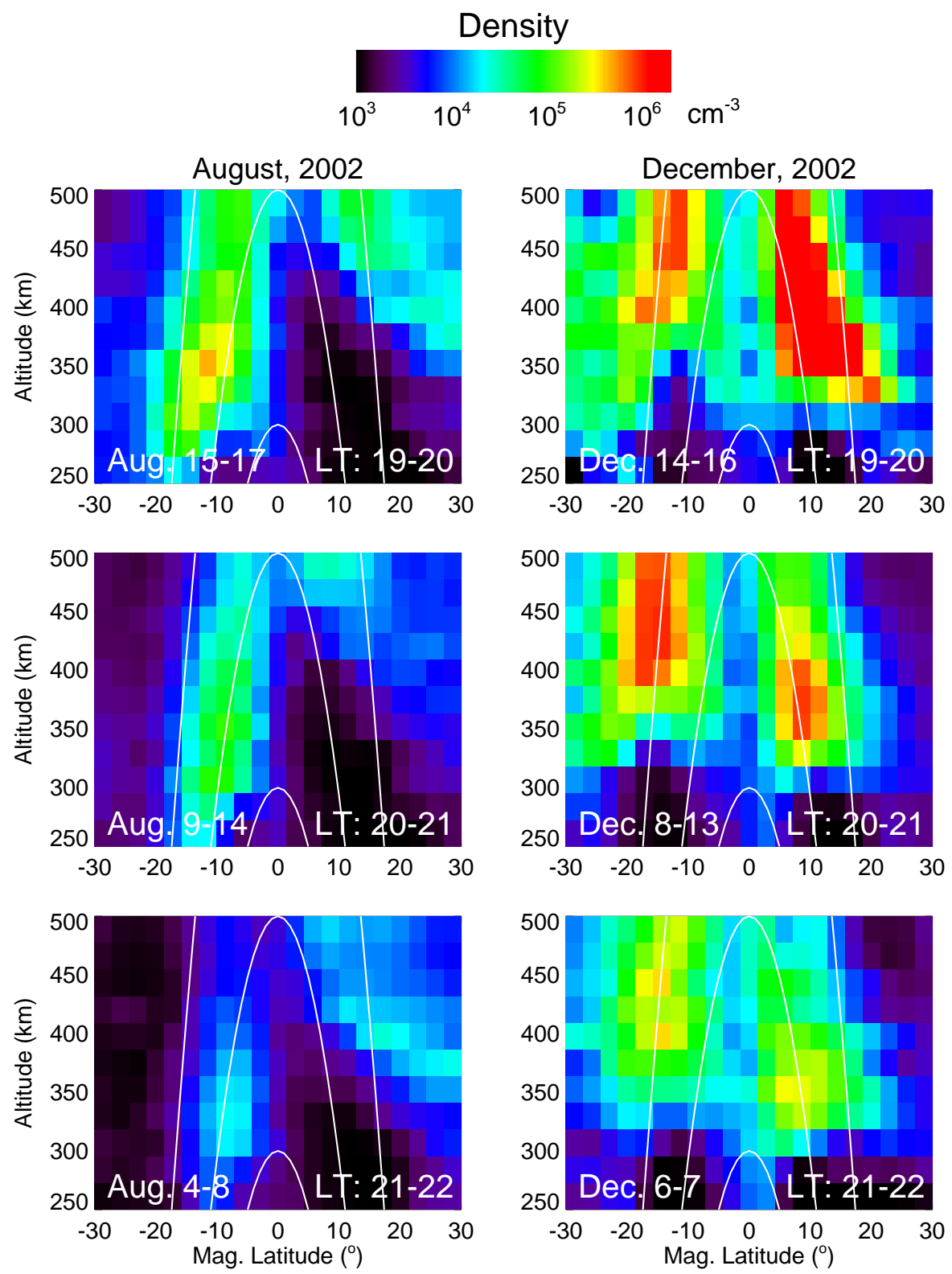

Fig. 2. Average electron density profiles deduced from the GUVI limb retrievals in the Atlantic sector during 4-17 August and 6-16 December 2002. The dipole field lines at the apex heights of $300 \mathrm{~km}, 500 \mathrm{~km}$, and $900 \mathrm{~km}$ are shown by white curves.

gyrofrequency to the collision frequency with neutral particles of the $j$-th species $\left(\Omega_{j} / v_{j n}\right)$. Here $\Omega_{j}=q_{j} B / m_{j}$ with $q_{j}$ and $m_{j}$ for the charge and mass of the $j$-th species, respectively. Above $130 \mathrm{~km}, 1 \ll \kappa_{i} \ll \kappa_{e}$, and $\sigma_{P}$ can be replaced by $\sigma_{P}=n m_{i} v_{i n} / B^{2}$. Here the ion-neutral collision frequency is given by

$v_{\text {in }}=\left(2.6 \times 10^{-9}\right)\left(\mathrm{n}_{\mathrm{n}}+\mathrm{n}\right) \mathrm{A}^{-1 / 2}$,

where $n_{n}$ is the neutral density per cubic centimeter, and A is the mean molecular weight of the neutrals and ions (Kelley, 1989). Since $\sigma_{P} \propto n\left(n_{n}+n\right), \Sigma_{P}^{F}$ or, consequently, the growth rate of the R-T instability increases with an increase in $F$-region plasma density. If $\Sigma_{P}^{F} \ll \Sigma_{P}^{E}$, then the growth rate becomes small and the bubble activity will be suppressed. The conducting electrons in the $E$-region short out the polarization electric field in the $F$ region. The $E$-region conductivity depends on the simultaneity of sunset at the conjugate points and therefore, the magnetic declination is the crucial factor. On the other hand, the $F$-region morphology, especially EIAs observed by GUVI, show large seasonal and longitudinal variations which cannot be explained by the magnetic field configuration. The uplift of the ionosphere to higher altitudes by the post-sunset enhancement of the eastward electric field is important for the growth of the 


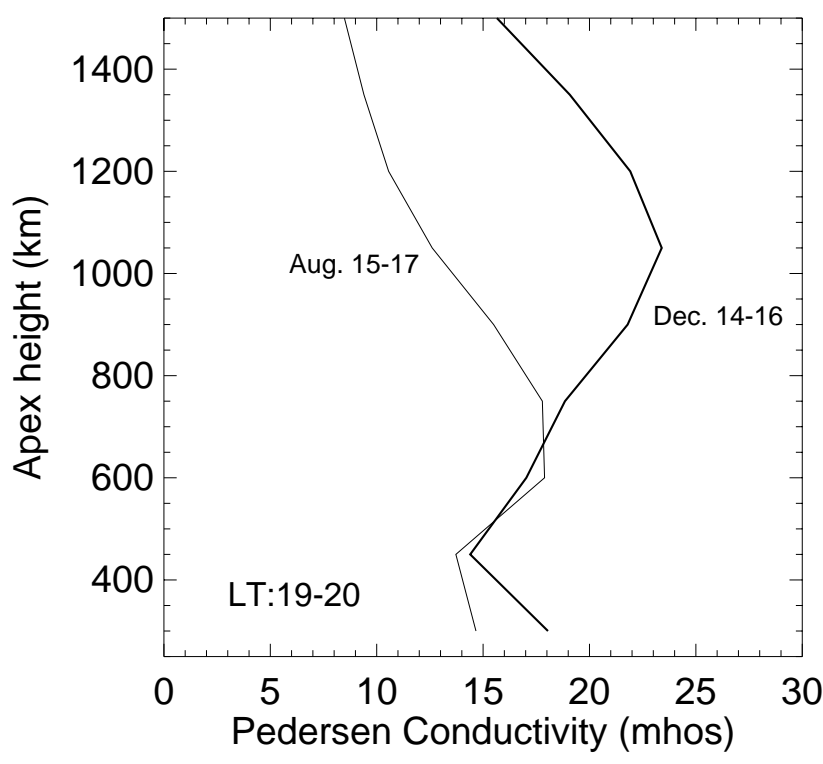

Fig. 3. $\Sigma_{P}^{F}$ at 19:00-20:00 LT in the Atlantic sector during 1517 August and 14-16 December 2002.

R-T instability through the influence on $V$ in Eq. (1). The other effect of the uplift of the ionosphere is the increase in $F$-region plasma density and consequently $\Sigma_{P}^{F}$. The redistribution of the $F$-region plasma by neutral winds also affects $\Sigma_{P}^{F}$. Therefore, $\Sigma_{P}^{F}$ is an $F$-region parameter that includes the effects of the electric fields and winds.

\section{Observations}

The occurrence of bubbles in regions of high background plasma density is often observed in the GUVI 135.6-nm images. Figure 1 shows the GUVI disk-scan images at 21:00 LT on 3-4 August (top), 6-7 December (middle), and 29-30 September (bottom) in 2002. Each image is the composite image of the 14 15 TIMED orbits of a day. The longitude and latitude bin size of the 135.6-nm radiance map is $0.5^{\circ} \times 0.5^{\circ}$. Emission of OI 135.6-nm from the nightside $F$ region is the result of a radiative recombination of $\mathrm{O}^{+}$ions with electrons and the subsequent decay of the excited states to the ground state. Since $\mathrm{O}^{+}$is the dominant $F$-layer constituent the OI 135.6-nm radiance is proportional to the lineof-sight integral of the square of the $F$-region electron density up to the satellite altitude. The $F$-region morphology may change day-to-day but some features occur repeatedly at the same longitude. The figure shows the smallest $135.6-\mathrm{nm}$ radiance in the Atlantic sector August and the strongest in this sector in December. Large longitudinal variations in 135.6$\mathrm{nm}$ radiance occur in in August and December. In September, the EIAs are well developed in both hemispheres and are distinguishable in most longitude regions. The narrow dark bands elongated in the north-south directions are produced by plasma depletions (e.g. Kelley et al., 2003; Kil et al., 2004b). The maps show the occurrence of the dark bands (plasma depletions) in the regions where the background radiance ( $F$-region plasma density) is large. Relatively uniform distribution of the bubbles in September may be associated with the development of strong EIAs in most longitude regions. These observations indicate the possible correlation of the bubble occurrence with the $F$-region plasma distribution.

To investigate the seasonal difference of the growth condition of bubbles we used the EDPs deduced from the GUVI limb retrievals (see DeMajistre et al. (2004) for the description of the GUVI limb retrieval). Precession of the TIMED satellite enables GUVI to sample the ionosphere for all local times every 60 days. Figure 2 shows average EDPs within a 1-h local time bin in the longitude range of $290^{\circ}-350^{\circ} \mathrm{E}$ during 4-17 August (left column) and 6-16 December (right column) in 2002. Each EDP at the given local time interval is obtained by averaging the EDPs of a few days. The dipole field lines at the apex heights of $300 \mathrm{~km}, 500 \mathrm{~km}$, and $900 \mathrm{~km}$ are shown by white lines. The altitude and latitude bin sizes of the EDPs are $25 \mathrm{~km}$ and $3^{\circ}$, respectively. The figure shows the presence of hemispheric asymmetries in the location and strength of the EIAs. As was shown in Fig. 1, the plasma density in August is much lower than that in December in the Atlantic sector. This phenomenon is observed consistently at different local times.

The equatorial plasma bubbles develop abruptly between 19:00-20:00 LT (e.g. Kil and Heelis, 1998). The decrease of the $E$-region conductivity, creation of a sharp plasma density gradient on the bottomside of the $F$ region, and uplift of the $F$ layer to higher altitudes provide a preferred condition for the growth of bubbles at 19:00-20:00 LT (Kelley, 1989). The flux-tube integrated $F$-region Pedersen conductivities are calculated using the EDPs in the Atlantic sector. In Fig. 3, we compare the seasonal difference of the $\Sigma_{P}^{F}$ at 19:00-20:00 LT, where $\Sigma_{P}^{F}$ at this time interval is calculated using the EDPs shown on the top panels of Fig. 2. The decrease in $\Sigma_{P}^{F}$ with apex height below $400 \mathrm{~km}$ is due to the decrease in plasma density and $\sigma_{P}$ with altitude. As the magnetic field lines intersect the EIAs, $\Sigma_{P}^{F}$ starts to increase above $400 \mathrm{~km}$. The difference in the strength and location of the EIAs in the two seasons causes the difference in the altitudinal $\Sigma_{P}^{F}$ profile above $700 \mathrm{~km}$. Stronger EIAs are developed at higher altitudes and latitudes in December than in August. As a result, $\Sigma_{P}^{F}$ further increases above $700 \mathrm{~km}$ in December. Therefore, bubbles can grow to higher altitudes in December than in August, if $\Sigma_{P}^{F}$ plays the dominant role for the growth of bubbles. In these calculations, the plasma distribution is assumed to drop off above $500 \mathrm{~km}$ with a constant scale height. However, the results are only weakly affected by this assumption, since the $F$ peak is usually located below $500 \mathrm{~km}$. In the future, the plasma density above $500 \mathrm{~km}$ can be extrapolated using the in-situ measurements 

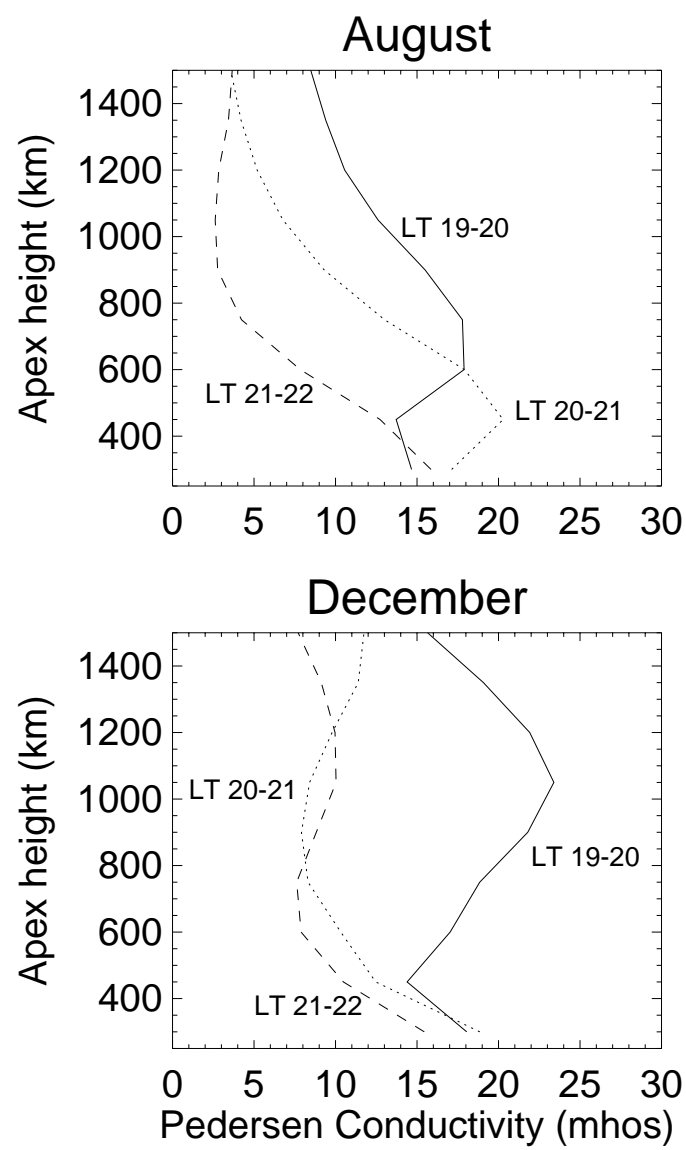

Fig. 4. Temporal evolution of the $\Sigma_{P}^{F}$.

from satellites or using a climatology for the rigorous calculation of the $\Sigma_{P}^{F}$.

We further investigate the local time variations of $\Sigma_{P}^{F}$. Figure 4 shows the decrease in $\Sigma_{P}^{F}$ at later local times. If we consider only the effect of the $F$ region on the growth of the bubbles, then the bubbles would preferentially develop at 19:00-20:00 LT rather than at later local times. We note that $\Sigma_{P}^{F}$ at different local times is calculated from data obtained on the different days, thus the day-to-day variability of the EIA may have an effect on our results. However, the physically reasonable decrease in $\Sigma_{P}^{F}$ at later local times is observed in both seasons, owing to the decrease in plasma density.

\section{Conclusions}

In an effort to understand the source mechanism of the global bubble distribution, we investigate the $F$-region effect on the growth of the R-T instability. The investigation is conducted in the Atlantic sector using the EDPs in August and December 2002, provided by the TIMED/GUVI. The $F$-region morphology shows a significant seasonal difference. The stronger EIAs are developed at higher altitudes and latitudes in December than the EIAs in August. As a result, the peak of $\Sigma_{P}^{F}$ occurs at a higher apex height in December than in August. At 19:00-20:00 LT, the peak value of $\Sigma_{P}^{F}$ has 23 mhos at $1100 \mathrm{~km}$ apex height during 14-16 December and $18 \mathrm{mhos}$ at $600 \mathrm{~km}$ during 15-17 August. Therefore, the $F$ region provides a preferred condition for the growth of bubbles to higher altitudes in December than in August. This study demonstrates the capability of using GUVI data for the calculation of $\Sigma_{P}^{F}$. Our future goal is to create the global maps of $\Sigma_{P}^{F}$ using the GUVI data. The comparison of the global $\Sigma_{P}^{F}$ maps with the global maps of the irregularities can be used to verify the importance of the $F$-region plasma distribution on the growth of bubbles.

Acknowledgements. H. Kil, R. DeMajistre, L. J. Paxton, and Y. Zhang acknowledge support from NASA TIMED program GUVI Grant NAG5-11412.

Topical Editor M. Pinnock thanks J. H. Sobral and another referee for their help in evaluating this paper.

\section{References}

Abdu, M. A., Bittencourt, J. A., and Batista, I. S.: Magnetic declination control of the equatorial $F$-region dynamo electric field development and spread $F$, J. Geophys. Res., 86, 11 443-11 446, 1981.

Abdu, M. A., Batista, I. S., and Sobral, J. H. A.: A new aspect of magnetic declination control of equatorial spread $F$ and $F$-region dynamo, J. Geophys. Res., 97, 14 897-14 904, 1992.

Christensen, A. B., Paxton, L. J., and Avery, S. et al.: Initial Observations with the Global Ultraviolet Imager (GUVI) in the NASA TIMED Satellite Mission, J. Geophys. Res., 108(A12), 1451, doi:10.1029/2003JA009918, 2003.

DeMajistre, R., Paxton, L. J., Morrison, D., Yee, J.-H., Goncharenko, L., and Christensen, A.: Retrievals of nighttime electron density from TIMED Global Ultraviolet Imager (GUVI) measurements, J. Geophys. Res., 109, A05305, doi:10.1029/2003JA010296, 2004.

Haerendel, G., Eccles, J. V., and Cakir, S.: Theory for modeling the equatorial evening ionosphere and the origin of the shear in the horizontal plasma flow, J. Geophys. Res., 97, 1209-1223, 1992.

Kelley, M. C.: The Earth's Ionosphere, Academic Press, San Diego, California, 1989.

Kil, H. and Heelis, R. A.: Global distribution of density irregularities in the equatorial ionosphere, J. Geophys. Res., 103, 407-417, 1998.

Kil, H., DeMajistre, R., and Paxton, L. J.:F-region plasma distribution seen from TIMED/GUVI and its relation to the equatorial spread $F$ activity, Geophys. Res. Lett., 31, L05810, doi:10.1029/2003GL018703, 2004a.

Kil, H., Su, S.-Y., Paxton, L. J., Wolven, B. C., Zhang, Y., Morrison, D., and Yeh, H. C.: Coincident equatorial bubble detection by TIMED/GUVI and ROCSAT-1, Geophys. Res. Lett., 31, L03809, doi:10.1029/2003GL018696, 2004b.

Maruyama, T. and Matuura, N.: Longitudinal variability of annual changes in activity of equatorial spread $F$ and plasma bubbles, J. Geophys. Res., 89, 10 903-10 912, 1984. 
McClure, J. P., Singh, S., Bamgboye, B. K., Johnson, F. S., and Kil, H.: Occurrence of equatorial $F$ region irregularities: Evidence for tropospheric seeding, J. Geophys. Res., 103, 29 119-29 135, 1998.

Mendillo, M., Baumgardner, J., Pi, X., and Sultan, P. J.: Onset condition for equatorial spread $F$, J. Geophys. Res., 97, 13 865$13876,1992$.

Paxton, L. J., Christensen, A. B., Morrison, D., Wolven, B., Kil, H., Zhang, Y., Ogorzalek, B. S., Humm, D. C., Goldsten, J., DeMajistre, R., and Meng, C. -I.: GUVI: A hyperspectral imager for geospace, Proc. SPIE, 5660, 227-240, doi:10.1117/12/579171, 2004.
Röttger, J.: Equatorial spread $F$ by electric fields and atmospheric gravity waves generated by thunderstorms, J. Atmos. Terr. Phys., 43, 453-462, 1981.

Sultan, P. J.: Linear theory and modeling of the Rayleigh-Taylor instability leading to the occurrence of equatorial spread $F$., J. Geophys. Res., 101, 26 875-26891, 1996.

Tsunoda, R. T.: Control of the seasonal and longitudinal occurrence of equatorial scintillations by the longitudinal gradient in integrated E region Pedersen conductivity, J. Geophys. Res., 90, 447-456, 1985. 